\title{
OPEN Pt and CoB trilayer Josephson $\pi$ junctions with perpendicular magnetic anisotropy
}

\author{
N. Satchell, T. Mitchell, P. M. Shepley, E. Darwin, B. J. Hickey \& G. Burnell ${ }^{\bowtie}$
}

We report on the electrical transport properties of $\mathrm{Nb}$ based Josephson junctions with $\mathrm{Pt} / \mathrm{Co}_{68} \mathrm{~B}_{32}$ /Pt ferromagnetic barriers. The barriers exhibit perpendicular magnetic anisotropy, which has the main advantage for potential applications over magnetisation in-plane systems of not affecting the Fraunhofer response of the junction. In addition, we report that there is no magnetic dead layer at the $\mathrm{Pt} / \mathrm{CO}_{68} \mathrm{~B}_{32}$ interfaces, allowing us to study barriers with ultra-thin $\mathrm{Co}_{68} \mathrm{~B}_{32}$. In the junctions, we observe that the magnitude of the critical current oscillates with increasing thickness of the $\mathrm{Co}_{68} \mathrm{~B}_{32}$ strong ferromagnetic alloy layer. The oscillations are attributed to the ground state phase difference across the junctions being modified from zero to $\pi$. The multiple oscillations in the thickness range $0.2 \leqslant d_{\mathrm{COB}} \leqslant 1.4 \mathrm{~nm}$ suggests that we have access to the first zero- $\pi$ and $\pi$-zero phase transitions. Our results fuel the development of low-temperature memory devices based on ferromagnetic Josephson junctions.

Proximity effects between superconducting $(S)$ and ferromagnetic $(F)$ materials are a topic of intense research effort due to the new physics at $S-F$ interfaces ${ }^{1-6}$. In $S-F-S$ Josephson junctions, it is well established that the ground-state phase difference across the junction can be tuned from zero to $\pi$, depending on the $F$ layer thickness ${ }^{1}$. Experimentally, the zero- $\pi$ transitions correspond to oscillations in the junction's characteristic voltage, $I_{c} R_{N}$, with increasing $F$ layer thickness ${ }^{7}$. To date, experimental demonstrations of $\pi$-junctions include: the weak ferromagnetic alloys $\mathrm{CuNi}^{8-14}, \mathrm{PdNi}^{15,16}$ and $\mathrm{PdFe}^{17}$, the ferromagnetic elements $\mathrm{Ni}^{18-26}, \mathrm{Co}^{20-22,27}$ and $\mathrm{Fe}^{20,21,28}$, and the strong ferromagnetic alloys $\mathrm{NiFe}^{20-22,29-32}, \mathrm{Ni}_{3} \mathrm{Al}^{33}, \mathrm{NiFeMo}^{34}$ and $\mathrm{NiFeCo}^{31}$.

In general, most previous works measure Josephson junctions with $F$ layers having in-plane magnetisation. When the magnetisation is in-plane, the $F$ layer can contribute significant magnetic flux density in the junction, modifying the response of the junction to an externally applied measurement field and shifting the maximum critical current away from $H=0$. In addition, when applying an in-plane field to measure the junction, the $F$ layer may switch in the measurement field. Josephson junctions containing perpendicular magnetic anisotropy (PMA) $F$ layers have advantages over in-plane systems as, in principle, the magnetisation and magnetic switching of layers in the junction should not affect the in-plane magnetic flux. The application of an in-plane measurement field will tilt the magnetisation of the PMA layer slightly, however the field required to fully saturate the PMA layers considered in this work is far larger than the field required to characterise the junctions, so the tilting effect can be neglected.

Of the previous $F$ layers characterised, only $\mathrm{CuNi}$ and PdNi have an intrinsic PMA component of their magnetisation. An alternative to intrinsic PMA is interfacial PMA, which can give a $F$ layer an overall PMA so long as the $F$ layer is thin enough that the interfacial anisotropy dominates over the bulk anisotropy. Josephson junctions containing interfacial PMA $F$ layers have been previously studied theoretically ${ }^{35-37}$ and experimentally in the context of spin-triplet supercurrents, however, no zero- $\pi$ oscillations were expected or observed in the particular geometries studied ${ }^{38-41}$.

In this work, we study the amorphous strong ferromagnetic alloy $\mathrm{Co}_{68} \mathrm{~B}_{32}{ }^{42}$. For many spintronic applications, the amorphous Co based alloys are advantageous over crystalline Co due to their lack of crystalline anisotropy and weaker pinning of magnetic domain walls due to the reduced density of grain boundaries ${ }^{43}$. Recently, thin film $\mathrm{Co}_{68} \mathrm{~B}_{32}$ has been studied for magnetic memory application and as a host of magnetic skyrmions ${ }^{44-46}$. When placed adjacent to Pt layers, the $\mathrm{Pt} / \mathrm{Co}_{68} \mathrm{~B}_{32}$ interfaces exhibit PMA, giving an overall PMA for the thin layers considered in this work. Previously, we used $\mathrm{Co}_{68} \mathrm{~B}_{32}$ in PMA pseudospin-valve junctions, where the critical current of the junction could be controlled by the relative orientation of two ferromagnets in the $\mathrm{Pt} / \mathrm{Co} / \mathrm{Pt} / \mathrm{Co}_{68}$ $\mathrm{B}_{32} / \mathrm{Pt}$ barrier ${ }^{47}$. For application in cryogenic memory, it is important to demonstrate that in addition to modulating the critical current of such devices, it is also possible to switch such devices from the zero to $\pi$ state ${ }^{48-51}$. 

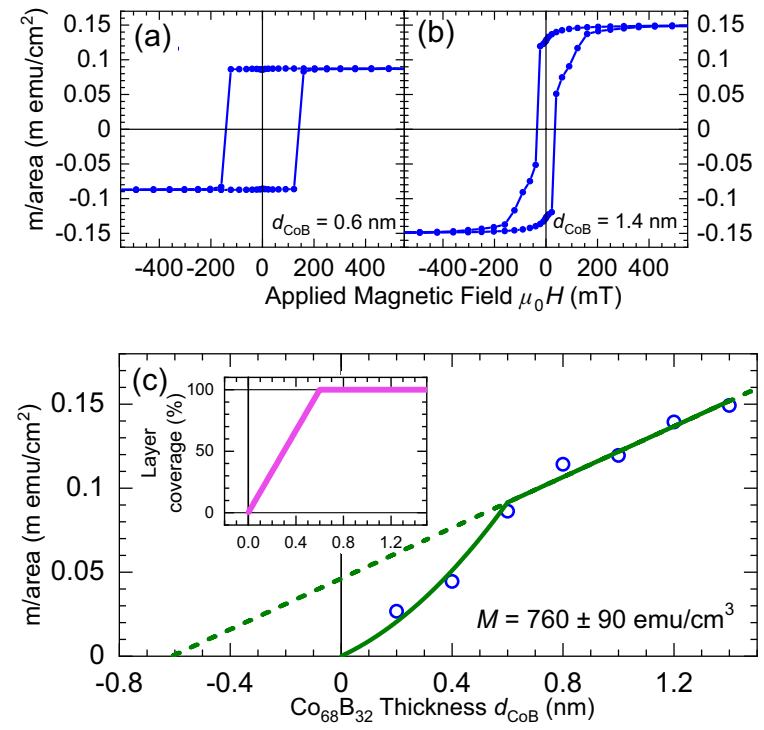

Figure 1. Magnetic characterisation of the sheet film samples $S$ - $\mathrm{Pt}(10)-\mathrm{Co}_{68} \mathrm{~B}_{32}\left(d_{\mathrm{CoB}}\right)-\mathrm{Pt}(5)-S$. (a,b) Magnetic hysteresis loops of the magnetic moment $(\mathrm{m})$ per area acquired at a temperature of $10 \mathrm{~K}$ with the applied field oriented out-of-plane for $(\mathbf{a}) d_{\mathrm{CoB}}=0.6 \mathrm{~nm}$ and $(\mathbf{b}) d_{\mathrm{CoB}}=1.4 \mathrm{~nm}$. The diamagnetic contribution from the substrate has been subtracted. (c) Collated saturation moment per area versus nominal thickness of $\mathrm{Co}_{68} \mathrm{~B}_{32}$. To model the lower $\mathrm{m} /$ area of the thinnest samples in the study, we construct a partial layer coverage model detailed in the text and shown in the inset. The result of fitting the model over the entire data range is shown by the solid line. The extracted magnetisation $M=760 \pm 90 \mathrm{emu} / \mathrm{cm}^{3}$. The dashed line shows an extrapolation of the linear part of the model to the intercepts. Values of $\mathrm{m} /$ area are calculated from the measured total magnetic moments and areas of the samples. The uncertainty in each point is dominated by the area measurements, and is less than $5 \%$.

For this, the zero- $\pi$ critical current oscillations of the component ferromagnets in the pseudospin-valve should be well characterised. In this work, we present evidence of such zero- $\pi$ critical current oscillations in $\mathrm{Pt} / \mathrm{Co}_{68}$ $\mathrm{B}_{32} /$ Pt junctions.

\section{Results}

Magnetic characterisation. Magnetic moment per area versus out-of-plane field data are shown in Fig. 1a,b for $S-\mathrm{Pt}(10)-\mathrm{Co}_{68} \mathrm{~B}_{32}\left(d_{\mathrm{CoB}}\right)-\mathrm{Pt}(5)-S$ sheet film samples at $10 \mathrm{~K}$ with a nominal thickness (a) $d_{\mathrm{CoB}}=0.6$ $\mathrm{nm}$ and (b) $d_{\mathrm{CoB}}=1.4 \mathrm{~nm}$. For $d_{\mathrm{CoB}}=0.6 \mathrm{~nm}$, the square hysteresis loop indicates a strong PMA. As the nominal thickness of the $\mathrm{Co}_{68} \mathrm{~B}_{32}$ is increased towards the largest thickness studied in this work, $d_{\mathrm{CoB}}=1.4$ nm Fig. 1b, we observe two changing characteristics in the hysteresis loops. Firstly, the coercive field reduces. Secondly, the squareness ratio of the loop reduces - indicating competing anisotropies in the $\mathrm{Co}_{68} \mathrm{~B}_{32}$ layer. Upon making the $\mathrm{CoB}$ thicker, we would expect that the anisotropy of the layer will change from being predominately PMA to predominately in-plane.

Saturation magnetic moment per area versus nominal thickness of the $\mathrm{Co}_{68} \mathrm{~B}_{32}\left(d_{\mathrm{CoB}}\right)$ at $10 \mathrm{~K}$ are shown in Fig. 1c. These data are best described in two regimes. For the thicker samples in this study, we observe the expected linear dependence with increasing thickness of ferromagnet. The thinnest two samples in this study deviate from this linear trend, showing a lower moment/area than implied by the trend in the thicker samples.

In order to apply a fitting model which describes the entire data set, we construct a partial layer coverage toy model of our system. The basis of the model is the assumption that the $F$-layers in the thinnest samples may not be continuous. From zero thickness to some critical thickness, we assume that the layer coverage increases linearly by the profile shown in Fig. 1c inset. The physical picture implied by this model is consistent with island nucleation, coalescence followed by layered growth - which is not an atypical growth mode for ambient temperature sputtered thin films. Above the critical thickness, we assume that the layer is now continuous, so the data can be described by the expected linear trend. More information on the model and extracting magnetisation from moment/area data are included in the Supplementary Information along with alternative fitting models to the data presented in Fig. 1c (see Supplementary Fig. S1 online).

The result of our toy model is shown by the solid line in Fig. 1c. The toy model gives a critical thickness for layer growth of $0.6 \pm 0.1 \mathrm{~nm}$, which is comparable to a couple of unit cells of nominal thickness, and gives the magnetisation of the $\mathrm{Co}_{68} \mathrm{~B}_{32}$ to be $M=760 \pm 90 \mathrm{emu} / \mathrm{cm}^{3}$, consistent with the expected bulk magnetisation of $730 \mathrm{emu} / \mathrm{cm}^{352}$. The model also includes a contribution to the total magnetic signal from the polarisation of the adjacent Pt layer, this is commonly observed in such systems ${ }^{53-57}$. In Figure 1 (c), the polarised Pt contribution can be extracted from the $y$-intercept of the dashed line, which is an extrapolation of the linear part of the 
(a)

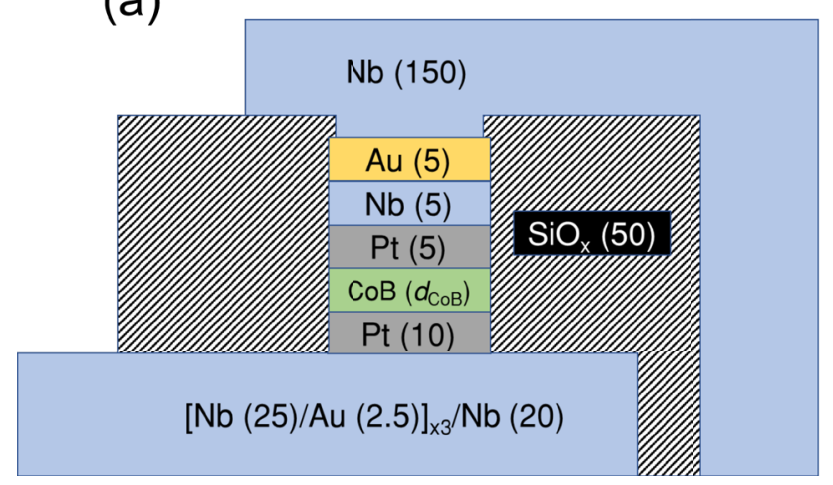

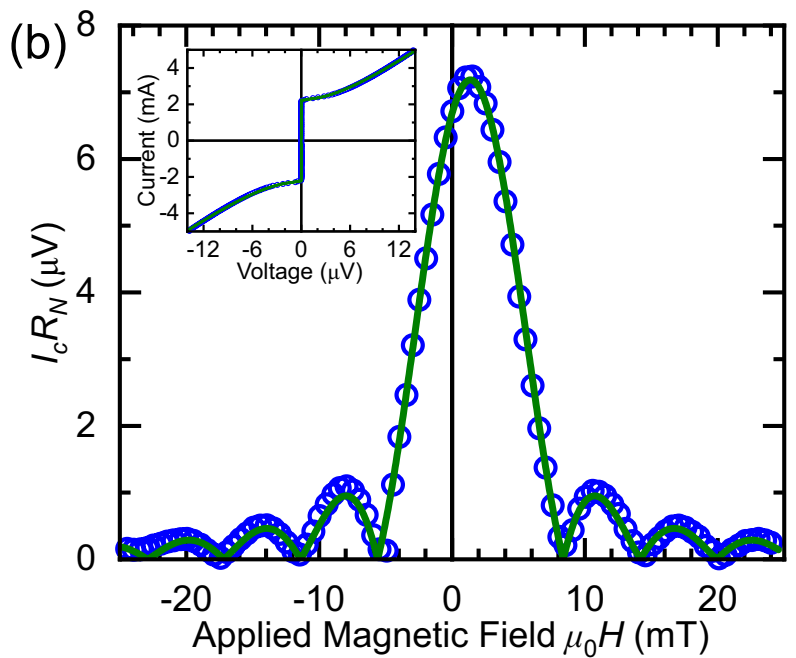

Figure 2. (a) Schematic cross section of the S-F-S Josephson junction device (not to scale). The thickness of each layer is given in nanometers. The $\mathrm{Co}_{68} \mathrm{~B}_{32}$ layer thickness, $d_{\mathrm{CoB}}$, is ranged from 0.2 to $1.4 \mathrm{~nm}$. (b) Product of critical Josephson current times normal-state resistance versus applied magnetic field for the device depicted in (a) with $d_{\mathrm{CoB}}=0.6 \mathrm{~nm}$ at $1.8 \mathrm{~K} . I_{c}$ is determined from the measured $I-V$ characteristic at each field value and $R_{N}$ is the average normal state resistance across all measured fields. The uncertainty in determining $I_{c} R_{N}$ is smaller than the data points. The data are fit with Eqs. (2) and (3). Inset: The $I-V$ characteristic at zero applied field with fit to Eq. (1).

model. The magnetic contribution to the total magnetic response of the sample by the polarised Pt is $46 \pm 9 \mu$ $\mathrm{emu} / \mathrm{cm}^{2}$, or $23 \pm 5 \mu \mathrm{emu} / \mathrm{cm}^{2}$ per $\mathrm{Pt} / \mathrm{Co}_{68} \mathrm{~B}_{32}$ interface, consistent with Suzuki et al. ${ }^{55}$.

It has been reported elsewhere that significant magnetic dead layers can form in ferromagnetic Josephson junction barriers at the $\mathrm{Nb} / F$ interfaces, see for example ${ }^{21}$, however adding buffer layers such as $\mathrm{Rh}$, $\mathrm{Cu}$ or Pt can significantly improve the morphology of the $F$ layer ${ }^{27,41,58,59}$. The signature of magnetic dead layers is a positive $\mathrm{x}$-intercept when plotting moment/area versus thickness. In our $\mathrm{Pt} / \mathrm{Co}_{68} \mathrm{~B}_{32} / \mathrm{Pt}$ barriers, the $\mathrm{x}$-intercept when fitting to Fig. 1c is not positive (regardless of the model used, see Supplementary Fig. S1 online), suggesting that such dead layers have been minimised by the Pt interfaces. Additionally, when the nominal thickness of the $\mathrm{Co}_{68}$ $\mathrm{B}_{32}$ is equivalent to only one or two monolayers, and so the layer is modelled with partial coverage, the polarised $\mathrm{Pt}$ appears to have stabilised the magnetisation of what we expect are islands of $\mathrm{Co}_{68} \mathrm{~B}_{32}$, allowing us to measure a magnetic response even for $d_{\mathrm{CoB}}=0.2 \mathrm{~nm}$, a significant advantage of our approach.

Electrical transport. Samples were fabricated using standard lithography techniques into circular current perpendicular-to-plane Josephson junction devices, as depicted schematically in Fig. 2a. We load the devices into our cryostat at room temperature and first cool to $15 \mathrm{~K}$, just above the superconducting transition ( $9 \mathrm{~K})$, where we apply a $1 \mathrm{~T}$ out-of-plane saturating field. Once the saturating field is removed, we rotate the sample so the field is applied in-plane and cool the samples to the base temperature of our cryostat, $1.8 \mathrm{~K}$. We measure the $I-V$ characteristic of each junction as a function of in-plane applied magnetic field between $\pm 25 \mathrm{mT}$ to determine the Fraunhofer pattern. The field necessary to saturate our $\mathrm{Pt} / \mathrm{Co}_{68} \mathrm{~B}_{32} / \mathrm{Pt}$ barriers is in excess of $1 \mathrm{~T}$ so the maximum deviation of the magnetisation from the perpendicular is less than $1.5^{\circ}$.

The $I-V$ characteristics of our devices follow the standard square-root form expected for over-damped Josephson junctions ${ }^{60}$,

$$
V=R_{N} \sqrt{I^{2}-I_{c}^{2}}, \text { for } I \geq I_{c}
$$

where $I_{c}$ is the critical Josephson current and $R_{N}$ is the normal state resistance of the junction. For circular Josephson junctions, the $I_{c}(B)$ Fraunhofer response can be described by the Airy function ${ }^{60}$,

$$
I_{c}=I_{c 0}\left|2 J_{1}\left(\pi \Phi / \Phi_{0}\right) /\left(\pi \Phi / \Phi_{0}\right)\right|,
$$

where $I_{c 0}$ is the maximum critical current, $J_{1}$ is a Bessel function of the first kind, $\Phi_{0}=h / 2 e$ is the flux quantum, and $\Phi$ is the flux through the junction ${ }^{60}$,

$$
\Phi=\mu_{0}\left(H_{\text {app }}-H_{\text {shift }}\right) w\left[\lambda_{\mathrm{L}}^{\text {bottom }} \tanh \left(d_{S}^{\text {bottom }} / 2 \lambda_{\mathrm{L}}^{\text {bottom }}\right)+\lambda_{\mathrm{L}}^{\text {top }} \tanh \left(d_{S}^{\text {top }} / 2 \lambda_{\mathrm{L}}^{\text {top }}\right)+d\right],
$$

where $w, \lambda_{\mathrm{L}}, d_{S}$, and $d$ are the width of the junction, the London penetration depth, the thickness of the superconducting electrode, and the total thickness of all the normal metal layers and $F$ layers in the junction, respectively. The bottom electrode is a Nb/Au multilayer $\left(\lambda_{\mathrm{L}}^{\text {bottom }}=190 \mathrm{~nm}^{61}\right)$ and the top electrode is single layer $\mathrm{Nb}$ $\left(\lambda_{\mathrm{L}}^{\text {top }}=150 \mathrm{~nm}^{62}\right) . H_{\text {app }}$ is the applied field and $H_{\text {shift }}$ is the amount $I_{c 0}$ is shifted from $H=0 . H_{\text {shift }}$ arises from a combination of an intrinsic contribution due to any in-plane magnetisation of the junction, and extrinsic artifacts 


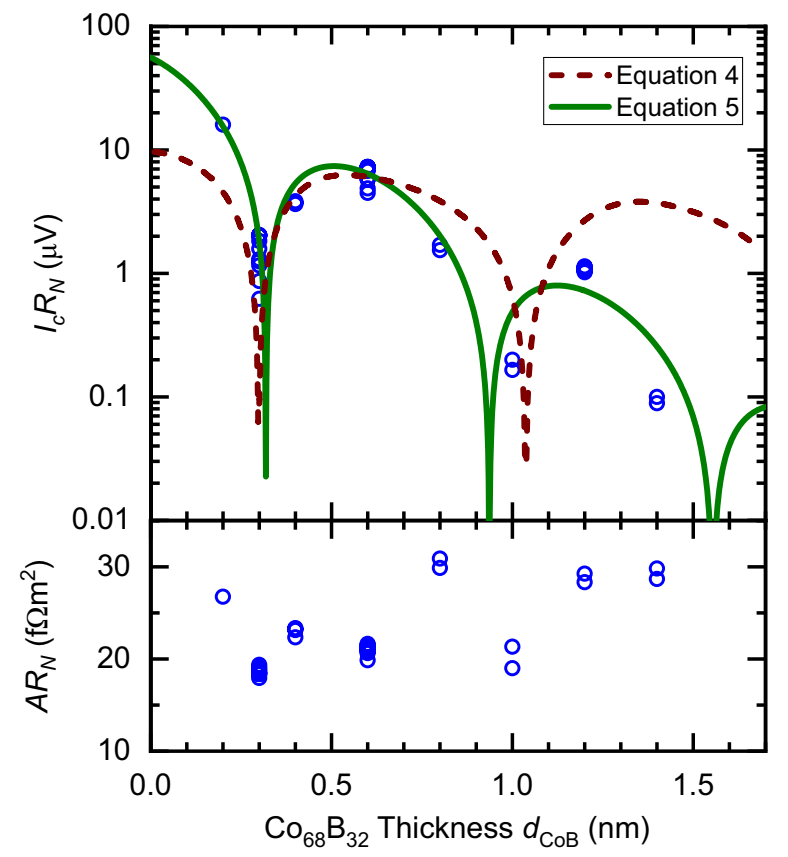

Figure 3. Top: Product of critical Josephson current times normal-state resistance versus nominal $\mathrm{Co}_{68}$ $\mathrm{B}_{32}$ thickness for ferromagnetic Josephson junctions of the form $S-\mathrm{Pt}(10)-\mathrm{Co}_{68} \mathrm{~B}_{32}\left(d_{\mathrm{CoB}}\right)-\mathrm{Pt}(5)-S$ at $1.8 \mathrm{~K}$. Each data point represents one Josephson junction and the uncertainty in determining $I_{c} R_{N}$ is smaller than the data points. The data are fit to Eqs. (4) and (5). The best fit parameters for Eq. (4) corresponds to $\xi_{F}=0.28 \pm 0.01 \mathrm{~nm}$, and for Eq. (5) to $\xi_{F 1}=0.28 \pm 0.02 \mathrm{~nm}$ and $\xi_{F 2}=0.20 \pm 0.02 \mathrm{~nm}$. The first minimum at $0.30 \pm 0.05 \mathrm{~nm}$ indicates a transition between the zero and $\pi$-phase states. Bottom: Product of the area times normal-state resistance for the same junctions. The scatter in $A R_{N}$ is most likely sample-to-sample variation in A.

from trapped flux in the $3 \mathrm{~T}$ superconducting coil used to perform the measurements. Fits to these equations are shown along with the data on a typical device in Fig. $2 \mathrm{~b}$. We attribute the small $H_{\text {shift }}$ in Fig. $2 \mathrm{~b}$ to trapped flux in our superconducting coil, which is supported by additional Fraunhofer data for both $+1 \mathrm{~T}$ and $-1 \mathrm{~T}$ saturation fields (see Supplementary Fig. S2 online). We determine $I_{c 0}$ for many samples of different $\mathrm{Co}_{68} \mathrm{~B}_{32}$ thicknesses $\left(d_{\mathrm{CoB}}\right)$ following the same protocol.

Figure 3 shows the collated $I_{c} R_{N}$ and $A R_{N}$ (area times normal-state resistance) products for the Josephson junctions measured in this study. $I_{c}$ corresponds to the $I_{c 0}$ maximum of the $I_{c}(B)$ Fraunhofer response and $R_{N}$ is the average resistance from measurements at all field values. As the thickness of the $\mathrm{Co}_{68} \mathrm{~B}_{32}$ is increased, the $I_{c} R_{N}$ shows nonmonotonic behaviour. When plotting $A R_{N}$, we fix $A$ by the nominal design dimension. The $A R_{N}$ product for our samples is suggestive that within the same chip the junction-to-junction reproducibility is very good, which is also supported by the small spread of $I_{c} R_{N}$ values for junctions on the same chip. It is possible to determine $A$ by fitting $I_{c}(B)$ to Eqs. (2) and (3), and we find that across all our junctions the extracted average $\bar{w}=3.0 \pm 0.3 \mu \mathrm{m}$ is consistent with the lithography design. The scatter in $A R_{N}$ is therefore similar to the scatter in the linear dimensions of the junctions. Variations in $A$ between samples will not affect the reported $I_{c} R_{N}$, which is a size independent quantity. Indeed, there is no correlation between a high/low $I_{c} R_{N}$ and $A R_{N}$.

We report strong reproduciblity of our results, as multiple samples for $d_{\mathrm{CoB}}=0.3$ and $0.6 \mathrm{~nm}$ are grown and fabricated in independent cycles and show consistency in $I_{c} R_{N}$, Fig. 3. Scatter in $I_{c} R_{N}$ is most likely driven by sample-to-sample variations in the thickness of the $\mathrm{Co}_{68} \mathrm{~B}_{32}$.

Coherence lengths in SIF/S Josephson junctions. The transport properties of $S-F-S$ Josephson junctions are well described in three limits, driven by the relative magnitude of three lengthscales; the mean free path $\left(l_{e}\right)$, the superconducting coherence length $\left(\xi_{S}\right)$ and the effective coherence length inside the ferromagnet $\left(\xi_{F}\right)$. In the ballistic limit $l_{e}>\xi_{S}>\xi_{F}$, in the intermediate limit $\xi_{S}>l_{e}>\xi_{F}$, and in the diffusive limit $\xi_{S}>\xi_{F}>l_{e}$.

In the ballistic limit, the decay and oscillations of $I_{\mathcal{C}} R_{N}$ is given by the numerical maximum with respect to $\varphi$ of the ballistic limit supercurrent $I_{\mathrm{S}}(\varphi)^{7}$,

$$
I_{\mathrm{S}}(\varphi) R_{N}=\frac{\pi \Delta \alpha^{2}}{2 e} \int_{\alpha}^{\infty} \frac{d y}{y^{3}}\left(\sin \frac{\varphi-y}{2} \tanh \frac{\Delta \cos \frac{\varphi-y}{2}}{2 k_{\mathrm{B}} T}+\sin \frac{\varphi+y}{2} \tanh \frac{\Delta \cos \frac{\varphi+y}{2}}{2 k_{\mathrm{B}} T}\right),
$$

where $\varphi$ is the phase difference across the junction, $\Delta$ is the energy gap, $T$ is the temperature, and $\alpha \equiv d / \xi_{F}$. In the ballistic limit, $\xi_{F}=\hbar v_{F} / 2 E_{\mathrm{Ex}}$, where $v_{F}$ is the Fermi velocity and $E_{\mathrm{Ex}}$ is the exchange energy. Ballistic limit 


\begin{tabular}{|c|c|c|c|c|c|}
\hline \multirow[b]{2}{*}{$F$} & \multirow{2}{*}{$\begin{array}{l}\xi_{F 1} \\
(\mathbf{n m})\end{array}$} & \multirow{2}{*}{$\begin{array}{l}\xi_{F 2} \\
(\mathbf{n m})\end{array}$} & \multirow{2}{*}{$\begin{array}{l}d_{\text {zero- } \pi} \\
(\mathrm{nm})\end{array}$} & \multirow{2}{*}{\begin{tabular}{|l|}
$V_{0}$ \\
$(\mu \mathrm{V})$ \\
\end{tabular}} & \multirow[b]{2}{*}{ References } \\
\hline & & & & & \\
\hline $\mathrm{Pd}_{97} \mathrm{Fe}_{3}$ & $16.2 \pm 1.4$ & $7.2 \pm 0.6$ & $16.3 \pm 0.2$ & $102 \pm 12$ & 17 \\
\hline $\mathrm{Ni}_{80} \mathrm{Fe}_{20}$ & $1.50 \pm 0.38$ & $0.58 \pm 0.10$ & $1.76 \pm 0.05$ & $69 \pm 19$ & 31 \\
\hline $\mathrm{Ni}_{65} \mathrm{Fe}_{15} \mathrm{Co}_{20}$ & $1.11 \pm 0.16$ & $0.48 \pm 0.03$ & $1.15 \pm 0.02$ & $30 \pm 6$ & 31 \\
\hline $\mathrm{Ni}_{73} \mathrm{Fe}_{21} \mathrm{Mo}_{6}$ & $0.48 \pm 0.04$ & $0.955 \pm 0.004$ & $2.25 \pm 0.10$ & $150 \pm 50$ & 34 \\
\hline $\mathrm{Co}_{68} \mathrm{~B}_{32}$ & $0.28 \pm 0.02$ & $0.20 \pm 0.02$ & $0.30 \pm 0.05$ & $56 \pm 8$ & This work \\
\hline
\end{tabular}

Table 1. Best fit parameters determined for selected $S-F-S$ Josephson junctions where the $F$ layers are ferromagnetic alloys and the $I_{c} R_{N}$ oscillations are well described by Eq. (5).

transport has been reported in the ferromagnetic elements when sufficiently thin, for example in Ni barriers studied by Robinson et al. ${ }^{20}$ and Baek et al..$^{25}$.

In the intermediate limit, the decay and oscillations of $I_{c} R_{N}$ is given by ${ }^{63}$,

$$
I_{c} R_{N}=V_{0} \exp \left(\frac{-d_{F}}{\xi_{F 1}}\right)\left|\sin \left(\frac{d_{F}-d_{\text {zero- } \pi}}{\xi_{F 2}}\right)\right|,
$$

where $d_{\text {zero- } \pi}$ is the thickness of the first zero- $\pi$ transition, $\xi_{F 1}=l_{e}$ and $\xi_{F 2}=\xi_{F}$ are the lengthscales governing the decay and oscillation of $I_{c} R_{N}$, respectively. In the intermediate limit, one finds $\xi_{F 1}>\xi_{F 2}$. Most ferromagnetic alloys studied in ferromagnetic Josephson junctions are solid-solutions with short mean free paths and are found to be best described in the intermediate limit ${ }^{17,31}$, for example PdNi barriers studied by Khaire et al. ${ }^{16}$.

It is well established that Rashba effects arising from spin-orbit coupling can be found at the interface between metallic ferromagnets, such as $\mathrm{Co}$, and heavy metals, such as $\mathrm{Pt}^{64-66}$ and so in the diffusive limit with spin-flip or spin-orbit scattering, the transport may be described by Eq. $(5)^{67}$. However in the diffusive limit one will find $\xi_{F 2}>\xi_{F 1}{ }^{67,68}$. In experimental literature, this situation is somewhat rarer, for example CuNi barriers studied by Oboznov et al. ${ }^{10}$ and NiFeMo barriers studied by Niedzielski et al. ${ }^{34}$.

Fitting to our results taking $\xi_{F}$ and $\xi_{F 1,2}$ as fitting parameters (shown in Fig. 3), we find that Eq. (4) does not reproduce our $\mathrm{Co}_{68} \mathrm{~B}_{32}$ data as well as Eq. (5), particularly for larger $d_{\mathrm{CoB}}$. The fits for Eq. (5) correspond to the limit $\xi_{F 1}>\xi_{F 2}$, placing our junctions in the intermediate limit. The best fit parameters are given in Table 1, which also includes results from other ferromagnetic alloys best described by Eq. (5). For further analysis on our data see Supplementary Fig. S3 and S4 online.

\section{Discussion}

Systems, such as ours, with a source of $s$-wave superconductivity, large spin-orbit coupling, and ferromagnetism are predicted to display transport properties consistent with spin-triplet supercurrents ${ }^{69}$, reported to be observed in ferromagnetic resonance measurements ${ }^{70}$. In this work, however, within the resolution of our measurements, the data are well described by singlet transport physics alone and we do not need to invoke a significant spintriplet supercurrent to explain our results - for further details see the Supplementary Information online. The lack of evidence for spin-triplet supercurrents in these Josephson junctions is consistent with previous works ${ }^{41,59}$.

In comparison to other ferromagnetic alloys, such as those in Table 1, our $\mathrm{Co}_{68} \mathrm{~B}_{32}$ junctions display significantly shorter $\xi_{F 1}$ and $\xi_{F 2}$ characteristic lengthscales. We attribute the short $\xi_{F 1}$ to the common property of amorphous alloys having a short $l_{e}$ due to structural disorder, however scattering at the $\mathrm{Pt} / \mathrm{Co}_{68} \mathrm{~B}_{32}$ interfaces may also be considerable for very thin $F$ layers. The advantage of the short $\xi_{F 2}$ in our system is that we have access to the first zero- $\pi$ and $\pi$-zero transitions before the $F$ layer undergoes the reorientation transition to in-plane magnetisation. Despite the short $\xi_{F 1}$, the extrapolated $I_{c} R_{N}$ at zero thickness for our junctions, $V_{0}=56 \pm 8 \mu \mathrm{V}$, is comparable to $\mathrm{Ni}_{80} \mathrm{Fe}_{20}$ junctions, $V_{0}=69 \pm 19 \mu \mathrm{V}^{31}$, which have been extensively studied for similar applications ${ }^{20-22,29-32,48-51}$. In addition, the $I_{c} R_{N}$ product at the peak of the first $\pi$ state, $V_{\pi} \approx 7 \mu \mathrm{V}$, is comparable to other ferromagnetic alloys, for example $V_{\pi} \approx 5 \mu \mathrm{V}$ in $\mathrm{Ni}_{65} \mathrm{Fe}_{15} \mathrm{Co}_{20}$ and $V_{\pi} \approx 12 \mu \mathrm{V}$ in $\mathrm{Ni}_{80} \mathrm{Fe}_{20}$ ${ }^{31}$. The disadvantage of the short $\xi_{F 2}$ for application is that precise control over thickness is necessary, since small variations will cause large changes to the $I_{c} R_{N}$ product and could potentially change the phase difference across the junction. Fortunately, such PMA multilayer stacks have an established industrial process for applications in magnetic recording.

The key application of ferromagnetic Josephson junctions is Josephson magnetic RAM (JMRAM) ${ }^{71}$. In this technology, the $\mathrm{Co}_{68} \mathrm{~B}_{32}$ forms one layer in a pseudospin-valve Josephson junction of the general type reported in our previous publication where we used $\mathrm{Co}$ and $\mathrm{Co}_{68} \mathrm{~B}_{32}{ }^{47}$. In order to use the pseudospin-valve for JMRAM, the component ferromagnetic layers must be well characterised, as we report here for $\mathrm{Pt} / \mathrm{Co}_{68} \mathrm{~B}_{32} / \mathrm{Pt} \mathrm{PMA}$ trilayers. In JMRAM, the zero- $\pi$ ferromagnetic junction is a passive phase shifter in a SQUID loop containing two $S / I / S$ junctions. As the zero- $\pi$ junction is passive, there is no need for this junction to have a large $I_{c} R_{N}$, however as we demonstrate our $\mathrm{Co}_{68} \mathrm{~B}_{32}$ has comparable performance in this regard to $\mathrm{Ni}_{80} \mathrm{Fe}_{20}$, which is used in state-of-theart devices. A JMRAM memory cell with in-plane ferromagnets $\left(\mathrm{Ni}_{80} \mathrm{Fe}_{20}\right.$ and $\left.\mathrm{Ni}\right)$ was recently demonstrated by Dayton et al..$^{50}$.

The detrimental features of magnetisation in-plane junctions are driven by the interaction between the physics underlying the Fraunhofer response and the vector potential of the ferromagnet. For in-plane single domain junctions, the Fraunhofer pattern will be uniformly shifted from zero global applied field by the vector potential 
of the ferromagnet in the plane of the junction. For example, Glick et al. characterise candidate in-plane $F$ layers ${ }^{31}$, where the Fraunhofer patterns are uniformly shifted by $H_{\text {shift }} \propto-M d_{F}$. To ensure that the $F$ layers are single domain, the maximum junction area in that work is $0.5 \mu \mathrm{m}^{2}$. If the area of the junctions is increased so that the ferromagnet becomes multidomain, it may not be possible to recover a Fraunhofer pattern due to the distortion by the stray fields emanating from the domains ${ }^{16,58,72}$. Such size considerations place an upper limit on the critical current of devices. Finally, the in-plane measurement field required to characterise the Fraunhofer pattern can cause premature switching of in-plane ferromagnets. Combined, the shift and premature switching means that JMRAM devices with in-plane ferromagnets may not have access to the highest critical current state, as observed for in-plane pseudospin-valve devices by Niedzielski et al. ${ }^{32}$.

In contrast, the use of PMA ferromagnets as we have demonstrated offers solutions to the issues introduced by in-plane ferromagnets. As the direction of the magnetisation and stray fields of PMA layers are parallel to the direction of current in the junction, they do not shift or distort the Fraunhofer pattern. Furthermore, due to the favourable demagnetisation effects, PMA materials systems have less stray fields and larger domain size limits compared to in-plane systems. Combined, these advantages allow us to successfully measure well defined Fraunhofer patterns on junctions with an area of $\approx 7 \mu \mathrm{m}^{2}$, much larger than those Glick et al. require to characterise in-plane layers. As a result of the PMA, the highest critical current state of the junction is available at zero global applied field, as demonstrated in Fig. 2b. Finally, the application of in-plane measurement fields will not switch a PMA ferromagnet.

For practicable memory devices, it will be important to remove the need for an applied out-of-plane switching field. Such a field to switch a PMA ferromagnet may introduce flux vortices into the superconducting $\mathrm{Nb}$ electrodes, which we negated in this work by applying such fields only above $T_{c}$. Routes towards removing this restriction by implementing an all electrical switching process include spin-transfer torque and spin-orbit torque switching of the barriers ${ }^{65,66,73}$.

\section{Conclusions}

In conclusion, we demonstrate Josephson $\pi$-junctions with $\mathrm{Pt} / \mathrm{Co}_{68} \mathrm{~B}_{32} / \mathrm{Pt}$ perpendicular magnetic anisotropy barriers. $\mathrm{Co}_{68} \mathrm{~B}_{32}$ is a strong ferromagnetic amorphous alloy of interest in spintronics due to its low pinning properties. We show that at the $\mathrm{Pt} / \mathrm{Co}_{68} \mathrm{~B}_{32}$ interfaces there is significant polarisation of the $\mathrm{Pt}$ and that the samples are magnetic down to a nominal $\mathrm{Co}_{68} \mathrm{~B}_{32}$ thickness of $0.2 \mathrm{~nm}$. In Josephson junctions, as the thickness of $\mathrm{Co}_{68} \mathrm{~B}_{32}$ is increased, we observe the nonmonotonic decay and oscillation of the critical Josephson current. These oscillations are attributed to the junctions undergoing the zero to $\pi$ transition. $\pi$-junctions have important applications in superconducting electronics, including cryogenic memory. Systematic material studies are crucial for the development of such technologies. The performance of our perpendicular magnetic anisotropy $\pi$-junctions are at least comparable to that of NiFe, which has in-plane magnetisation.

\section{Methods}

Samples are deposited, fabricated, and measured using identical methodology to our previous work ${ }^{47}$. The final product of cleanroom processing are standard "sandwich" planar Josephson junctions, defined by photolithography and $\mathrm{Ar}^{+}$ion milling, where the current flows perpendicular to the plane. The diameter of the circular junctions is a design parameter and is nominally $3 \mu \mathrm{m}$.

We dc sputter deposit the multilayer samples onto thermally oxidised Si substrates in the Royce Deposition System ${ }^{74}$. The magnetrons are mounted below, and confocal to, the substrate with source-substrate distances of $134 \mathrm{~mm}$. The base pressure of the vacuum chamber is $1 \times 10^{-9} \mathrm{mbar}$. The samples are deposited at room temperature with an $\mathrm{Ar}\left(6 \mathrm{~N}\right.$ purity) gas pressure of $3.6 \times 10^{-3} \mathrm{mbar}$ for the $[\mathrm{Nb} / \mathrm{Au}]_{\mathrm{x} 3} / \mathrm{Nb}$ bottom electrode layers and $4.8 \times 10^{-3} \mathrm{mbar}$ for the $\mathrm{Pt} / \mathrm{Co}_{68} \mathrm{~B}_{32} / \mathrm{Pt}$ barrier layers. The $[\mathrm{Nb} / \mathrm{Au}]_{\mathrm{x} 3} / \mathrm{Nb}$ superlattice is used for the bottom electrode as the superlattice has a lower surface roughness compared to a single Nb layer of comparable total thickness ${ }^{61,75}$. Finally, a Nb/Au cap is deposited to prevent oxidation during the processing. In the final stage of sample fabrication, the top electrode, $150 \mathrm{~nm}$ of Nb, is deposited after an in-situ ion milling process to remove $5 \mathrm{~nm}$ from the $10 \mathrm{~nm}$ Au cap. The full structure of the final device with thickness in $(\mathrm{nm})$ is $[\mathrm{Nb}(25) / \mathrm{Au}(2.5)]_{\mathrm{x} 3}$ $/ \mathrm{Nb}(20) / \mathrm{Pt}(10) / \mathrm{Co}_{68} \mathrm{~B}_{32}\left(d_{\mathrm{CoB}}\right) / \mathrm{Pt}(5) / \mathrm{Nb}(5) / \mathrm{Au}(5) / \mathrm{Nb}(150)$.

The choice of $\mathrm{Pt}$ thicknesses in this study is informed by previous results ${ }^{41}$, being a balance between developing a suitable textured surface on which to grow the $\mathrm{Co}_{68} \mathrm{~B}_{32}$ layer whilst not being in a range that significantly affects the critical current density. The total thickness of the Pt in this work is the same as that required for pseudospin-valve devices ${ }^{47}$.

Fabricated devices are measured in a continuous flow ${ }^{4} \mathrm{He}$ cryostat with $3 \mathrm{~T}$ horizontal superconducting Helmholtz coils. The sample can be rotated about the vertical axis, which we perform in increments of $90^{\circ}$ to bring that field in- and out-of-plane of the junctions. In order to avoid trapping flux in the superconducting $\mathrm{Nb}$ layers in the devices, we performed all sample rotations in zero field above the $T_{c}$ of the $\mathrm{Nb}$ and always cooled the sample in zero applied field (in practice there will inevitably be a small remanent field due to trapped flux in the magnet). The full sequence of setting the magnetic state of our samples and performing the measurement field sweeps is: warm to $15 \mathrm{~K}$, rotate sample to apply field out-of-plane, apply saturating field, remove saturating field, rotate sample by $90^{\circ}$, cool sample, apply field in-plane, measure. Once we have finished measuring that magnetic state of the sample, we may wish to measure a further condition, such as reversing the magnetisation. To do so, we remove the in-plane field, warm to $15 \mathrm{~K}$ and repeat the cycle described above. 
Traditional 4-point-probe transport geometry is used to measure the current-voltage characteristic of the junction with combined Keithley 6221-2182A current source and nano-voltmeter. Magnetisation loops of sheet films are measured using a Quantum Design MPMS 3 magnetometer.

\section{Data availability}

The datasets generated during the current study are available in the University of Leeds repository, https://doi. org/10.5518/817.

Received: 1 September 2020; Accepted: 5 April 2021

Published online: 27 May 2021

\section{References}

1. Buzdin, A. I. Proximity effects in superconductor-ferromagnet heterostructures. Rev. Mod. Phys. 77, 935-976. https://doi.org/10. 1103/RevModPhys.77.935 (2005).

2. Bergeret, F. S., Volkov, A. F. \& Efetov, K. B. Odd triplet superconductivity and related phenomena in superconductor-ferromagnet structures. Rev. Mod. Phys. 77, 1321-1373. https://doi.org/10.1103/RevModPhys.77.1321 (2005).

3. Eschrig, M. Spin-polarized supercurrents for spintronics. Phys. Today 64, 43-49. https://doi.org/10.1063/1.3541944 (2011).

4. Linder, J. \& Robinson, J. W. A. Superconducting spintronics. Nat. Phys. 11, 307-315. https://doi.org/10.1038/nphys3242 (2015).

5. Eschrig, M. Spin-polarized supercurrents for spintronics: a review of current progress. Rep. Prog. Phys. 78, 104501. https://doi. org/10.1088/0034-4885/78/10/104501 (2015).

6. Birge, N. O. Spin-triplet supercurrents in Josephson junctions containing strong ferromagnetic materials. Philos. Trans. R. Soc. A 376, 20150150. https://doi.org/10.1098/rsta.2015.0150 (2018).

7. Buzdin, A. I., Bulaevskii, L. N. \& Panyukov, S. V. Critical-current oscillations as a function of the exchange field and thickness of the ferromagnetic metal (F) in an SFS Josephson junction. JETP Lett. 35, 178-180 (1982).

8. Ryazanov, V. V. et al. Coupling of Two Superconductors through a Ferromagnet: evidence for a $\pi$ Junction. Phys. Rev. Lett. 86, 2427-2430. https://doi.org/10.1103/PhysRevLett.86.2427 (2001).

9. Sellier, H., Baraduc, C., Lefloch, F. \& Calemczuk, R. Temperature-induced crossover between 0 and $\pi$ states in S/F/S junctions. Phys. Rev. B 68, 054531. https://doi.org/10.1103/PhysRevB.68.054531 (2003).

10. Oboznov, V. A., Bol'ginov, V. V., Feofanov, A. K., Ryazanov, V. V. \& Buzdin, A. I. Thickness dependence of the Josephson ground states of superconductor-ferromagnet-superconductor junctions. Phys. Rev. Lett. 96, 197003. https://doi.org/10.1103/PhysRevLett. 96.197003 (2006).

11. Weides, M. et al.0- $\pi$ Josephson tunnel junctions with ferromagnetic barrier. Phys. Rev. Lett. 97, 247001. https://doi.org/10.1103/ PhysRevLett.97.247001 (2006).

12. Weides, M. et al. High quality ferromagnetic 0 and $\pi$ Josephson tunnel junctions. Appl. Phys. Lett. 89, 122511. https://doi.org/10. $1063 / 1.2356104(2006)$

13. Stoutimore, M. J. A. et al. Second-harmonic current-phase relation in Josephson junctions with ferromagnetic barriers. Phys. Rev. Lett. 121, 177702. https://doi.org/10.1103/PhysRevLett.121.177702 (2018).

14. Bolginov, V. V., Rossolenko, A. N., Shkarin, A. B., Oboznov, V. A. \& Ryazanov, V. V. Fabrication of Optimized superconducting phase inverters based on superconductor-ferromagnet-superconductor $\pi$-junctions. J. Low Temp. Phys. 190, 302-314. https://doi. org/10.1007/s10909-017-1843-6 (2018).

15. Kontos, T. et al. Josephson junction through a thin ferromagnetic layer: negative coupling. Phys. Rev. Lett. 89, 137007. https://doi. org/10.1103/PhysRevLett.89.137007 (2002).

16. Khaire, T. S., Pratt, W. P. \& Birge, N. O. Critical current behavior in Josephson junctions with the weak ferromagnet PdNi. Phys. Rev. B 79, 094523. https://doi.org/10.1103/PhysRevB.79.094523 (2009).

17. Glick, J. A., Loloee, R., Pratt, W. P. \& Birge, N. O. Critical current oscillations of Josephson junctions containing PdFe nanomagnets. IEEE Trans. Appl. Supercond. 27, 1-5. https://doi.org/10.1109/TASC.2016.2630024 (2017).

18. Blum, Y., Tsukernik, A., Karpovski, M. \& Palevski, A. Oscillations of the superconducting critical current in Nb-Cu-Ni-Cu-Nb junctions. Phys. Rev. Lett. 89, 187004. https://doi.org/10.1103/PhysRevLett.89.187004 (2002).

19. Shelukhin, V. et al. Observation of periodic $\pi$-phase shifts in ferromagnet-superconductor multilayers. Phys. Rev. B 73, 174506. https://doi.org/10.1103/PhysRevB.73.174506 (2006).

20. Robinson, J. W. A., Piano, S., Burnell, G., Bell, C. \& Blamire, M. G. Critical current oscillations in strong ferromagnetic $\pi$ junctions. Phys. Rev. Lett. 97, 177003. https://doi.org/10.1103/PhysRevLett.97.177003 (2006).

21. Robinson, J. W. A., Piano, S., Burnell, G., Bell, C. \& Blamire, M. G. Zero to $\pi$ transition in superconductor-ferromagnet-superconductor junctions. Phys. Rev. B 76, 094522. https://doi.org/10.1103/PhysRevB.76.094522 (2007).

22. Robinson, J. W. A., Piano, S., Burnell, G., Bell, C. \& Blamire, M. G. Transport and magnetic properties of strong ferromagnetic pi-junctions. IEEE Trans. Appl. Supercond. 17, 641-644. https://doi.org/10.1109/TASC.2007.898720 (2007).

23. Bannykh, A. A. et al. Josephson tunnel junctions with a strong ferromagnetic interlayer. Phys. Rev. B 79, 054501. https://doi.org/ 10.1103/PhysRevB.79.054501 (2009).

24. Baek, B., Rippard, W. H., Benz, S. P., Russek, S. E. \& Dresselhaus, P. D. Hybrid superconducting-magnetic memory device using competing order parameters. Nat. Commun. 5, 3888. https://doi.org/10.1038/ncomms4888 (2014).

25. Baek, B., Schneider, M. L., Pufall, M. R. \& Rippard, W. H. Phase offsets in the critical-current oscillations of Josephson junctions based on $\mathrm{Ni}$ and Ni-( $\left.\mathrm{Ni}_{81} \mathrm{Fe}_{19}\right)_{x} \mathrm{Nb}_{y}$ barriers. Phys. Rev. Appl. 7, 064013. https://doi.org/10.1103/PhysRevApplied.7.064013 (2017).

26. Baek, B., Schneider, M. L., Pufall, M. R. \& Rippard, W. H. Anomalous supercurrent modulation in Josephson junctions with Nibased barriers. IEEE Trans. Appl. Supercond. 28, 1-5. https://doi.org/10.1109/TASC.2018.2836961 (2018).

27. Robinson, J. W. A., Barber, Z. H. \& Blamire, M. G. Strong ferromagnetic Josephson devices with optimized magnetism. Appl. Phys. Lett. 95, 192509. https://doi.org/10.1063/1.3262969 (2009).

28. Piano, S., Robinson, J. W. A., Burnell, G. \& Blamire, M. G. 0- $\pi$ oscillations in nanostructured Nb/Fe/Nb Josephson junctions. Eur. Phys. J. B 58, 123-126. https://doi.org/10.1140/epjb/e2007-00210-8 (2007).

29. Bell, C., Loloee, R., Burnell, G. \& Blamire, M. G. Characteristics of strong ferromagnetic Josephson junctions with epitaxial barriers. Phys. Rev. B 71, 180501(R). https://doi.org/10.1103/PhysRevB.71.180501 (2005).

30. Abd El Qader, M. et al. Switching at small magnetic fields in Josephson junctions fabricated with ferromagnetic barrier layers. Appl. Phys. Lett. 104, 022602. https://doi.org/10.1063/1.4862195 (2014).

31. Glick, J. A. et al. Critical current oscillations of elliptical Josephson junctions with single-domain ferromagnetic layers. J. Appl. Phys. 122, 133906. https://doi.org/10.1063/1.4989392 (2017).

32. Niedzielski, B. M. et al. Spin-valve Josephson junctions for cryogenic memory. Phys. Rev. B 97, 024517. https://doi.org/10.1103/ PhysRevB.97.024517 (2018).

33. Born, F. et al. Multiple 0- $\pi$ transitions in superconductor/insulator/ferromagnet/superconductor Josephson tunnel junctions. Phys. Rev. B 74, 140501(R). https://doi.org/10.1103/PhysRevB.74.140501 (2006). 
34. Niedzielski, B. M., Gingrich, E. C., Loloee, R., Pratt, W. P. \& Birge, N. O. S/F/S Josephson junctions with single-domain ferromagnets for memory applications. Supercond. Sci. Technol. 28, 085012. https://doi.org/10.1088/0953-2048/28/8/085012 (2015).

35. Margaris, I., Paltoglou, V. \& Flytzanis, N. Zero phase difference supercurrent in ferromagnetic Josephson junctions. J. Phys. Condens. Matter 22, 445701. https://doi.org/10.1088/0953-8984/22/44/445701 (2010).

36. Halterman, K., Valls, O. T. \& Wu, C.-T. Charge and spin currents in ferromagnetic Josephson junctions. Phys. Rev. B 92, 174516. https://doi.org/10.1103/PhysRevB.92.174516 (2015).

37. Silaev, M. A., Tokatly, I. V. \& Bergeret, F. S. Anomalous current in diffusive ferromagnetic Josephson junctions. Phys. Rev. B 95, 184508. https://doi.org/10.1103/PhysRevB.95.184508 (2017).

38. Gingrich, E. C. et al. Spin-triplet supercurrent in $\mathrm{Co} / \mathrm{Ni}$ multilayer Josephson junctions with perpendicular anisotropy. Phys. Rev. B 86, 224506. https://doi.org/10.1103/PhysRevB.86.224506 (2012).

39. Glick, J. A. et al. Spin-triplet supercurrent in Josephson junctions containing a synthetic antiferromagnet with perpendicular magnetic anisotropy. Phys. Rev. B 96, 224515. https://doi.org/10.1103/PhysRevB.96.224515 (2017).

40. Glick, J. A. et al. Phase control in a spin-triplet SQUID. Sci. Adv. 4, eaat9457. https://doi.org/10.1126/sciadvaat9457 (2018).

41. Satchell, N., Loloee, R. \& Birge, N. O. Supercurrent in ferromagnetic Josephson junctions with heavy-metal interlayers. II. Canted magnetization. Phys. Rev. B 99, 174519. https://doi.org/10.1103/PhysRevB.99.174519 (2019).

42. Tanaka, H. et al. Electronic structure and magnetism of amorphous $\mathrm{Co}_{1-x} \mathrm{~B}_{x}$ alloys. Phys. Rev. B 47, 2671-2677. https://doi.org/ 10.1103/PhysRevB.47.2671 (1993).

43. Lavrijsen, R. et al. Reduced domain wall pinning in ultrathin $\mathrm{Pt} / \mathrm{Co}_{100-x} \mathrm{~B} x / \mathrm{Pt}$ with perpendicular magnetic anisotropy.. Appl. Phys. Lett. 96, 022501. https://doi.org/10.1063/1.3280373 (2010).

44. Schellekens, A. J., Van den Brink, A., Franken, J. H., Swagten, H. J. M. \& Koopmans, B. Electric-field control of domain wall motion in perpendicularly magnetized materials. Nat. Commun. 3, 847. https://doi.org/10.1038/ncomms1848 (2012).

45. Finizio, S. et al. Deterministic field-free Skyrmion nucleation at a nanoengineered injector device. Nano Lett. 19, 7246-7255. https://doi.org/10.1021/acs.nanolett.9b02840 (2019).

46. Zeissler, K. et al. Diameter-independent skyrmion Hall angle observed in chiral magnetic multilayers. Nat. Commun. 11, 428. https://doi.org/10.1038/s41467-019-14232-9 (2020).

47. Satchell, N. et al. Spin-valve Josephson junctions with perpendicular magnetic anisotropy for cryogenic memory. Appl. Phys. Lett. 116, 022601. https://doi.org/10.1063/1.5140095 (2020).

48. Bell, C. et al. Controllable Josephson current through a pseudospin-valve structure. Appl. Phys. Lett. 84, 1153-1155. https://doi. org/10.1063/1.1646217 (2004).

49. Gingrich, E. C. et al. Controllable 0- $\pi$ Josephson junctions containing a ferromagnetic spin valve. Nat. Phys. 12, 564. https://doi. org/10.1038/nphys3681 (2016).

50. Dayton, I. M. et al. Experimental demonstration of a Josephson magnetic memory cell with a programmable $\pi$-junction. IEEE Magn. Lett. 9, 3301905. https://doi.org/10.1109/LMAG.2018.2801820 (2018).

51. Madden, A. E., Willard, J. C., Loloee, R. \& Birge, N. O. Phase controllable Josephson junctions for cryogenic memory. Supercond. Sci. Technol. 32, 015001. https://doi.org/10.1088/1361-6668/aae8cf (2018).

52. Konč, M. et al. Temperature dependence of the magnetization and of the other physical properties of rapidly quenched amorphous CoB alloys. IEEE Trans. Magn. 30, 524-526. https://doi.org/10.1109/20.312324 (1994).

53. Schütz, G. et al. Spin-dependent X-ray absorption in Co/Pt multilayers and $\mathrm{Co}_{50} \mathrm{Pt}_{50}$ alloy. J. Appl. Phys. 67, 4456-4458. https:// doi.org/10.1063/1.344903 (1990).

54. Geissler, J. et al. Pt magnetization profile in a Pt/Co bilayer studied by resonant magnetic X-ray reflectometry. Phys. Rev. B 65, 020405(R). https://doi.org/10.1103/PhysRevB.65.020405 (2001).

55. Suzuki, M. et al. Depth profile of spin and orbital magnetic moments in a subnanometer Pt film on Co.. Phys. Rev. B 72, 054430. https://doi.org/10.1103/PhysRevB.72.054430 (2005).

56. Rowan-Robinson, R. M. et al. The interfacial nature of proximity-induced magnetism and the Dzyaloshinskii-Moriya interaction at the Pt/Co interface. Sci. Rep. 7, 16835. https://doi.org/10.1038/s41598-017-17137-z (2017).

57. Inyang, O. et al. Threshold interface magnetization required to induce magnetic proximity effect. Phys. Rev. B 100, 174418. https:// doi.org/10.1103/PhysRevB.100.174418 (2019).

58. Khasawneh, M. A., Pratt, W. P. \& Birge, N. O. Josephson junctions with a synthetic antiferromagnetic interlayer. Phys. Rev. B 80, 020506(R). https://doi.org/10.1103/PhysRevB.80.020506 (2009).

59. Satchell, N. \& Birge, N. O. Supercurrent in ferromagnetic Josephson junctions with heavy metal interlayers. Phys. Rev. B 97, 214509. https://doi.org/10.1103/PhysRevB.97.214509 (2018).

60. Barone, A. \& Paternò, G. Physics and Applications of the Josephson Effect (Wiley, 1982).

61. Quarterman, P. et al. Distortions to the penetration depth and coherence length of superconductor/normal-metal superlattices. Phys. Rev. Mater. 4, 074801. https://doi.org/10.1103/PhysRevMaterials.4.074801 (2020).

62. Flokstra, M. G. et al. Observation of Anomalous Meissner Screening in $\mathrm{Cu} / \mathrm{Nb}$ and $\mathrm{Cu} / \mathrm{Nb} / \mathrm{Co}$ Thin Films. Phys. Rev. Lett. 120, 247001. https://doi.org/10.1103/PhysRevLett.120.247001 (2018).

63. Bergeret, F. S., Volkov, A. F. \& Efetov, K. B. Josephson current in superconductor-ferromagnet structures with a nonhomogeneous magnetization. Phys. Rev. B 64, 134506. https://doi.org/10.1103/PhysRevB.64.134506 (2001).

64. Krupin, O. et al. Rashba effect at magnetic metal surfaces. Phys. Rev. B 71, 201403. https://doi.org/10.1103/PhysRevB.71.201403 (2005).

65. Miron, I. M. et al. Current-driven spin torque induced by the Rashba effect in a ferromagnetic metal layer. Nat. Mater. 9, $230-234$. https://doi.org/10.1038/nmat2613 (2010).

66. Miron, I. M. et al. Perpendicular switching of a single ferromagnetic layer induced by in-plane current injection. Nature 476, 189. https://doi.org/10.1038/nature10309 (2011).

67. Fauré, M., Buzdin, A. I., Golubov, A. A. \& Kupriyanov, M. Y. Properties of superconductor/ferromagnet structures with spindependent scattering. Phys. Rev. B 73, 064505. https://doi.org/10.1103/PhysRevB.73.064505 (2006).

68. Pugach, N. G., Kupriyanov, M. Y., Goldobin, E., Kleiner, R. \& Koelle, D. Superconductor-insulator-ferromagnet-superconductor Josephson junction: from the dirty to the clean limit. Phys. Rev. B 84, 144513. https://doi.org/10.1103/PhysRevB.84.144513 (2011).

69. Bergeret, F. S. \& Tokatly, I. V. Spin-orbit coupling as a source of long-range triplet proximity effect in superconductor-ferromagnet hybrid structures. Phys. Rev. B 89, 134517. https://doi.org/10.1103/PhysRevB.89.134517 (2014).

70. Jeon, K.-R. et al. Enhanced spin pumping into superconductors provides evidence for superconducting pure spin currents. Nat. Mater. 17, 499-503. https://doi.org/10.1038/s41563-018-0058-9 (2018).

71. Herr, A. Y. \& Herr, Q. P. Josephson magnetic random access memory system and method (2012). US Patent 8270209.

72. Bourgeois, O. et al. Josephson effect through a ferromagnetic layer. Eur. Phys. J. B 21, 75-80. https://doi.org/10.1007/s100510170 $215(2001)$

73. Baek, B. et al. Spin-transfer torque switching in nanopillar superconducting-magnetic hybrid Josephson junctions. Phys. Rev. Appl. 3, 011001. https://doi.org/10.1103/PhysRevApplied.3.011001 (2015).

74. The Royce Deposition System is a multi-chamber, multi-technique thin film deposition tool based at the University of Leeds as part of the Henry Royce Institute.

75. Wang, Y., Pratt, W. P. \& Birge, N. O. Area-dependence of spin-triplet supercurrent in ferromagnetic Josephson junctions. Phys. Rev. B 85, 214522. https://doi.org/10.1103/PhysRevB.85.214522 (2012). 


\section{Acknowledgements}

We wish to thank Norman Birge for advice and helpful discussions, M. Vaughan, J. Massey, M. Rogers, T. Moorsom, M. Ali, M. Rosamond, and L. Chen for experimental assistance. We acknowledge support from the Henry Royce Institute. The work was supported financially through the following EPSRC grants: EP/M000923/1, $\mathrm{EP} / \mathrm{P} 022464 / 1$ and EP/R00661X/1. This project has received funding from the European Unions Horizon 2020 research and innovation programme under the Marie Skłodowska-Curie Grant Agreement No. 743791 (SUPERSPIN).

\section{Author contributions}

N.S. and G.B. conceived and designed the experiment. N.S., P.M.S., E.D., B.J.H. and G.B. undertook the materials development including optimisation and characterisation of the growth process. N.S., T.M. and G.B. undertook the low temperature transport and magnetometry measurements and analysed the data. N.S. undertook the sample fabrication and wrote the manuscript. All authors reviewed and edited the manuscript.

\section{Competing interests}

The authors declare no competing interests.

\section{Additional information}

Supplementary Information The online version contains supplementary material available at https://doi.org/ 10.1038/s41598-021-90432-y.

Correspondence and requests for materials should be addressed to G.B.

Reprints and permissions information is available at www.nature.com/reprints.

Publisher's note Springer Nature remains neutral with regard to jurisdictional claims in published maps and institutional affiliations.

(c) (i) Open Access This article is licensed under a Creative Commons Attribution 4.0 International License, which permits use, sharing, adaptation, distribution and reproduction in any medium or format, as long as you give appropriate credit to the original author(s) and the source, provide a link to the Creative Commons licence, and indicate if changes were made. The images or other third party material in this article are included in the article's Creative Commons licence, unless indicated otherwise in a credit line to the material. If material is not included in the article's Creative Commons licence and your intended use is not permitted by statutory regulation or exceeds the permitted use, you will need to obtain permission directly from the copyright holder. To view a copy of this licence, visit http://creativecommons.org/licenses/by/4.0/.

(C) The Author(s) 2021 